\title{
NÍVEIS DE CORTISOL SALIVAR E ESTRESSE EM INDIVÍDUOS COM LÍQUEN PLANO BUCAL: UM ESTUDO DE CASO CONTROLE
}

\author{
Izana Santos Borges Nascimento ${ }^{1}$; Ynara Bosco de Oliveira Lima-Arsati; Alessandra \\ Laís Pinho Valente Pires ${ }^{3}$ e Valéria Souza Freitas ${ }^{4}$
}

\author{
1. Bolsista PIBIC/CNPq, Graduando em Odontologia, Universidade Estadual de Feira de Santana, e-mail: \\ izana_nascimento@yahoo.com.br \\ 2. Orientador, Departamento de Ciências Biológicas, Universidade Estadual de Feira de Santana, e-mail: ynara@uefs.br \\ 3. Participante do NUCAO - Núcleo de Câncer Oral, aluna de Doutorado em Saúde Coletiva, Departamento de Saúde, \\ Universidade Estadual de Feira de Santana, e-mail: lecavalent@ hotmail.com \\ 4. Coordenadora do NUCAO - Núcleo de Câncer Oral, Departamento de Saúde, Universidade Estadual de Feira de Santana, \\ e-mail: valeria.souza.freitas@gmail.com
}

PALAVRAS-CHAVE: cortisol salivar; estresse; líquen plano bucal.

\section{INTRODUÇÃO}

O Líquen Plano (LP) é uma doença mucocutânea inflamatória crônica, de etiologia incerta, que pode afetar pele e ou mucosas, sendo a mais comum das alterações dermatológicas com manifestações bucais, quando é chamado de Líquen Plano Bucal (LPB) (Ma et al., 2013; Payeras et al., 2013).

Estudos têm sido realizados para investigar os fatores capazes de desencadear ou agravar o LPB, com especial atenção aos fatores psicológicos (Vallejo et al. 2001; Lundqvist et al. 2006; Silva et al. 2007; Tawil, Sediki, Hassan 2009; Scattarella et al. 2011). Como estes fatores não são facilmente mensuráveis, algumas pesquisas têm utilizado testes psicológicos buscando quantificar e comprovar a relação entre o LPB e alterações comportamentais, visto que indivíduos com a doença geralmente apresentam-se mais estressados (Soto Araya, Rojas, Esguep, 2004; Gavic et al. 2014; Sandhu et al. 2014). Assim, pretende-se com este estudo avaliar o estresse e o padrão de secreção do cortisol salivar em indivíduos com LPB, a fim de compreender a relação entre a doença e este fator psicológico.

\section{MATERIAL E MÉTODOS}

Um estudo caso-controle foi conduzido no Centro de Referência em Lesões Bucais (CRLB) da Universidade Estadual de Feira de Santana (UEFS), após aprovação do Comitê de Ética em Seres Humanos desta Instituição (CAAE: 05590612.7.0000.0053; parecer 114.132).

O grupo de casos foi composto por 21 indivíduos com diagnóstico clínico/histopatológico de líquen plano bucal. O grupo controle, proveniente da mesma população de origem dos casos, foi constituído por 21 indivíduos sem a doença, admitidos de forma aleatória e pareados por sexo e idade em relação aos casos. Os participantes foram submetidos a exame clínico da cavidade bucal, entrevista estruturada, teste psicológico (Escala de Estresse Percebido- EPP) e coleta de saliva em três momentos distintos.

Os dados foram analisados, inicialmente, por meio de estatística descritiva. $\mathrm{O}$ teste de Mann-Whitney foi aplicado para comparar os grupos com relação aos escores de estresse e o padrão de secreção do cortisol salivar. O nível de significância adotado foi de $5 \%$.

\section{RESULTADOS E/OU DISCUSSÃO}

A população do estudo foi constituída por 42 indivíduos. A maioria era mulheres $(71,4 \%)$, apresentava idade acima de quarenta anos $(69,0 \%)$, com média de idade de 48,64 $( \pm 13,73)$ anos. As formas clínicas, reticular e em placa, foram predominantes $(85,71 \%)$ e, a atrófica e erosiva, foram observadas em 14,29\% dos indivíduos. As lesões variaram em diversos sítios da cavidade bucal, acometendo com predileção a mucosa jugal $(52,38 \%)$. 
Quando aplicado a EEP, foi observada diferença significativa entre os grupos quanto aos escores de estresse $(\mathrm{p}=0,026)$. Em acréscimo, verificou-se no nosso estudo uma chance quatro vezes maior $(\mathrm{OR}=4,0)$ de os indivíduos com $\mathrm{LPB}$ estarem expostos ao estresse (Tabela 1). Lundqvist et al. (2006) constatou, além da depressão e ansiedade, uma forte correlação entre LPB e estresse. No estudo de Čanković, Bokor-Bratić e Novović (2015), revelou-se que, a cada nova experiência estressante, aumenta-se em quase duas vezes a chance de desenvolver LPB (OR de 1,97; $\mathrm{p}<0,001)$.

Tabela 1. Distribuição dos indivíduos dos indivíduos com LPB e controles, odds ratio (OR), intervalo de confiança (IC 95\%), quanto à presença de estresse.

\begin{tabular}{lcccccc}
\hline \multicolumn{1}{c}{ Variável } & \multicolumn{7}{c}{ Casos } & \multicolumn{2}{c}{ Controles } \\
& $\begin{array}{c}\mathrm{N} \\
(\mathrm{n}=21)\end{array}$ & $\%$ & $\mathrm{~N}$ & $\%$ & OR & IC (95\%) \\
& & & & & & \\
\hline Estresse percebido & 15 & 71,42 & 6 & 39,13 & & $1,11-14,80$ \\
$\begin{array}{l}\text { Presença de estresse } \\
\text { Ausência de estresse }\end{array}$ & 8 & 38,08 & 13 & 60,87 & 4,0 & \\
\hline
\end{tabular}

Alguns estudos utilizaram biomarcadores salivares buscando obter padrões mais objetivos de mensuração de fatores psicológicos (Rödström et al., 2001; Mutsuura et al., 2009; Girardi et al., 2011;Vrshek-Schallhorn et al., 2013; Shah et al., 2015). Quanto às concentrações de cortisol salivar, em ambos os grupos houve uma variação ao longo do dia, entre as três coletas (A, B e C). Foram calculados também os valores de Resposta do Cortisol ao Acordar $(\mathrm{RCA}=\mathrm{B}-\mathrm{A})$ e Declínio diurno $(\mathrm{DD}=\mathrm{B}-\mathrm{C})$. Não houve diferença estatisticamente significativa nos valores médios da RCA e DD (Tabela 2).

Tabela 2. Concentração do padrão de cortisol salivar ( $\mu \mathrm{g} / \mathrm{dL})$ nos indivíduos com LPB e controles, Feira de Santana, 2014-2016.

\begin{tabular}{lccr}
\hline \multicolumn{1}{c}{ Variáveis } & $\begin{array}{c}\text { Grupo caso } \\
\text { Média }( \pm \mathrm{DP})\end{array}$ & $\begin{array}{c}\text { Grupo controle } \\
\text { Média }( \pm \mathrm{DP})\end{array}$ & $\mathrm{p}^{*}$ \\
\hline Ao acordar (A) & $0,30( \pm 0,12)$ & $0,30( \pm 0,15)$ & 0,98 \\
30 minutos após acordar $(\mathrm{B})$ & $0,37( \pm 0,21)$ & $0,33( \pm 0,14)$ & 0,95 \\
Antes de dormir $(\mathrm{C})$ & $0,06( \pm 0,06)$ & $0,09( \pm 0,09)$ & 0,97 \\
RCA (B-A) & $0,08( \pm 0,25)$ & $0,02( \pm 0,17)$ & 0,37 \\
Declínio diurno (B $-\mathrm{C})$ & $0,3( \pm 0,23)$ & $0,24( \pm 0,21)$ & 0,38 \\
\hline
\end{tabular}

Ao se comparar o padrão de secreção do cortisol salivar entre os tempos de coleta não se verificou diferença estatisticamente significante da concentração deste hormônio, assim como evidenciado por Rodstrom et al., (2001), Girard et al. (2011), Pippi et al. (2014) Contrapondo tais achados, Koray et al., (2003) verificaram níveis de cortisol salivar significativamente superiores em indivíduos com LPB. Entretanto, nesse estudo foi realizada apenas uma coleta de saliva antes do procedimento de biópsia nos indivíduos avaliados, o que pode ter induzido o aumento do estresse e consequente elevação da secreção de cortisol. Shah, Ashok \& Sujatha (2015) reportam maiores níveis de estresse, ansiedade, depressão e cortisol em pacientes com LPB. Nadendla et al., (2014) também observaram níveis maiores de cortisol salivar em indivíduos com LPB e concluíram que o fator psicológico desempenha num papel importante na patogênese da doença. 
Diferenças nos resultados encontrados em relação ao padrão de secreção do cortisol salivar nos estudos sobre LPB podem estar relacionadas ao tamanho das amostras, ao uso de diferentes metodologias com variações quanto ao protocolo de coleta e dosagem do cortisol, bem como em relação aos critérios para a inclusão dos casos, limitando, dessa forma, a generalização e comparação dos dados, de acordo com Girard et al. (2011).

\section{CONSIDERAÇÕES FINAIS}

De acordo com os resultados obtidos no presente estudo foi demonstrada uma associação significativa do estresse percebido nos indivíduos com líquen plano bucal, embora o fluxo salivar e o padrão de secreção de cortisol salivar não diferiram entre os indivíduos com a doença e os controles. A tendência de baixos níveis de cortisol salivar encontrado em indivíduos com LPB podem ser resultantes de alterações inflamatórias locais que culminam com aumento da atuação do eixo HHA. Tais achados indicam a necessidade de investigação de novos biomarcadores, envolvendo outros eixos do sistema nervoso autônomo, que possam melhor refletir a resposta ao estresse nos indivíduos com LPB.

\section{REFERÊNCIAS}

ČANKOVIĆ, M. Bokor-Bratić M, Novović Z. 2015.Stressful life events and personality traits in patients with oral lichen planus. Acta Dermatovener Cr.; 23(4):270-6.

GIRARDI, C.et al. .2011.Salivary cortisol and dehydroepiandrosterones (DHEA) levels, psychological factors in patients with oral lichen planus. Archives Oral Biology, v. 56, n. 9, p. 864-868.

KORAY, M. et al. 2003.The evaluation of anxiety and salivary cortisol levels in patients with oral lichen planus. Oral diseases, v. 9, n. 6, p. 298-301.

LUNDQVIST, E et al. 2006.Psychological health in patients with genital and oral erosive lichen planus. Journal of the European Academy of Dermatology and Venereology,v. 20, n. 6, p. 661666.

MA, L. et al. 2013.Bmi1 expression in oral lichen planus and the risk of progression to oral squamous cell carcinoma. Annals of Diagnostic Pathology, v. 17, n. 4, p. 327-330.

NADENDLA et al. 2014.Salivary Cortisol and Anxiety levels in lichen planus. Patients. Journal of Clinical and Diagnostic Research, v. 8, n. 12,p. ZC01-ZC03.

PAYERAS, M. R. et al. 2013.Oral lichen planus: focus on etiopathogenesis. Archives of Oral Biology, v. 58, n. 9, p. 1057-1069.

PIPPI, R. et al.2014.Diurnal trajectories of salivary cortisol, salivary $\alpha$-amylase and psychological profiles in oral lichen planus patients. Journal of Biological Regulators \& Homeostatic Agents,v. 28 , n. 1 , p. 147-54.

RÖDSTRÖM, PO et al. 2001. Erosive oral lichen planus and salivary cortisol. J Oral Pathol Med.; 30(5): p. 257-263.

SANDHU S V et al. 2014. Oral lichen planus and stress: An appraisal. Contemp. Clin. Dent.; 5(3): 352-356.

SOTO ARAYA M.; ROJAS ALCAYAGA G.; ESGUEP A. 2004.Association between psychological disorders and the presence of Oral lichen planus, Burning mouth syndrome and Recurrent aphthous stomatitis. Medicine Oral, v. 9, n.1, p. 1-7. 
SHAH B, ASHOK L, SUJATHA G P. 2015.Evaluation of salivary cortisol and psychological factors in patients with oral lichen planus. Indian J Dent Res. v.20, n. 3, p. 288-292.

TAWIL M EL, Sediki N, 2009. Hassan H. Psychobiological Aspects of Patients with Lichen Planus. Current Psychiatry.;16(4): 370-380.

VALLEJO, M.J.G-P. et al. 2001. Anxiety and depression as risk factors for oral lichen planus. Dermatology.; 203(4): p. 303-307. 